\title{
Nanoparticles in biomedicine: Let there be intrinsic light
}

\author{
Tomljenovic-Hanic $\mathrm{S}^{1 *}$, Connor $\mathrm{AJO}^{2}$ and Morrison $\mathrm{WA}^{3,4}$ \\ ${ }^{1}$ School of Physics, University of Melbourne, Australia \\ ${ }^{2}$ Department of Chemical and Biomolecular Engineering, University of Melbourne, Australia \\ ${ }^{3}$ O'Brien Institute, St Vincent's Institute of Medical Research, Australia \\ ${ }^{4}$ Departmnet of Surgery, St Vincent's Hospital, University of Melbourne, Australia
}

\begin{abstract}
In this commentary we point out that metal oxide and semi-metallic nanoparticles can be tracked by their own fluorescence without need to use dyes and proteins for tagging. The presence of fluorescent agents modifies the chemistry of the sample and that may be responsible for induced changes in the properties being monitored. Therefore, it is highly advantageous if these nanoparticles intrinsic fluorescence can be used for tracking without any additional tags. In this way, there are two essential advantages. One is that there is no induced change of the chemistry or dynamics of these nanoparticles. Second, we point out that their fluorescence differs from the cell's' auto-fluorescence. We believe that this comment which discusses the intrinsic fluorescence of these nanoparticles would be important for researches in biomedicine, nanobiotechnology and nanoscale bioimaging.
\end{abstract}

\section{Commentary}

In the last few years, the study of metal oxide and semi-metallic nanoparticles (NPs) has become a distinctive subject of research. This increase in interest is driven by the numerous applications of these materials in industry, medicine, information technology, energy storage, sensing and many others [1,2]. Most importantly, they are used as an additive to human and animal food and in biomedical applications.

Their fluorescence has been widely investigated within materials science and it also has widespread use in industrial applications. One example is zinc oxide $(\mathrm{ZnO})$, whose fluorescence has been studied for decades. It exhibits a strong emission peak in the UV, due to recombination of free excitons, and broadband emission in the visible region, attributed to defects within the crystallographic structure [3]. Another example is semi-metallic selenium (Se), with the largest worldwide usage in glass manufacturing, due to its red and pink color [4].

The therapeutic properties of these nanoparticles (NPs) have been extensively investigated for many pharmaceutical and biomedical applications. The therapeutic properties of $\mathrm{ZnO}$ have been well explored as anti-cancer and antibacterial agents $[5,6]$. Selenium is a trace element, naturally found in the body. Recent studies point out that Se has the potential to affect human health in many ways, including improving thyroid function, cancer prevention, capture of heavy metals and antibacterial function for use in orthopaedic applications [4].

However, in biomedical research, their intrinsic fluorescence has been rarely used for particle tracking. Biomedical scientists are commonly using commercial confocal microscopies that use dyes and proteins for tagging, usually not aware of these NPs' autofluorescence. The fluorescent agents, used in commercial confocals, modify the chemistry of the sample and they may be inducing changes in the properties being monitored such as particle transport. Additionally, for tracking purposes single photon sensitive scanning confocal fluorescence imaging is not necessary but it is a big advantage as measurement is not limited by concentration [3].
The main challenge for optical imaging probes is to satisfy all the criteria necessary for in vitro and in vivo applications. These are: biocompatibility, biodegradability, photostability, suitable wavelengths of absorbance, and fluorescence that differs from tissue autofluorescence. It is well known that in a biological cell, the presence of components such as collagens and flavins produces fluorescent background signals with the absorption band in the range $300-500 \mathrm{~nm}$ and the emission band in the range $400-550 \mathrm{~nm}$. Therefore, it is essential for the NPs to absorb light at wavelengths longer than $500 \mathrm{~nm}$ and to emit light at wavelengths longer than $600 \mathrm{~nm}$ in order to be effectively detected and tracked in the cellular environment [7]. Additionally, the light emission should be in the near-infrared region (NIR), 700-900 $\mathrm{nm}$, as it penetrates centimeters into tissue, whereas visible light can only travel microns [8].

Organic dyes and fluorescent proteins meet some of these requirements, but they suffer from photobleaching [9]. Quantum dots (QDs) have been investigated extensively due to their brightness but the major obstacle in the clinical use of QDs remains their toxicity [10]. In contrast, optical centres in nanodiamond (ND) are increasingly being viewed as the crucial building blocks for the development of a variety of advanced biotechnologies due to their outstanding photostability, biocompatibility and suitable wavelengths of absorbance and fluorescence, however NDs do not biodegrade [11] (Figure 1).

Previously, intrinsic fluorescence from metal oxide has been reported as unstable, and that was the main obstacle for their usage in bioimaging application $[3,12]$. Several metal oxide dopants were

${ }^{\star}$ Correspondence to: SnjezanaTomljenovic-Hanic, Department of Chemical and Biomolecular Engineering, University of Melbourne, VIC 3010, Australia, Tel: +61 38344 5094; Fax: +61 39347 4783; E-mail: snjezana.thanic@unimelb.edu.au

Key words: nanoparticles, fluorescence, bioimaging

Received: April 02, 2018; Accepted: April 20, 2018; Published: April 25, 2018 


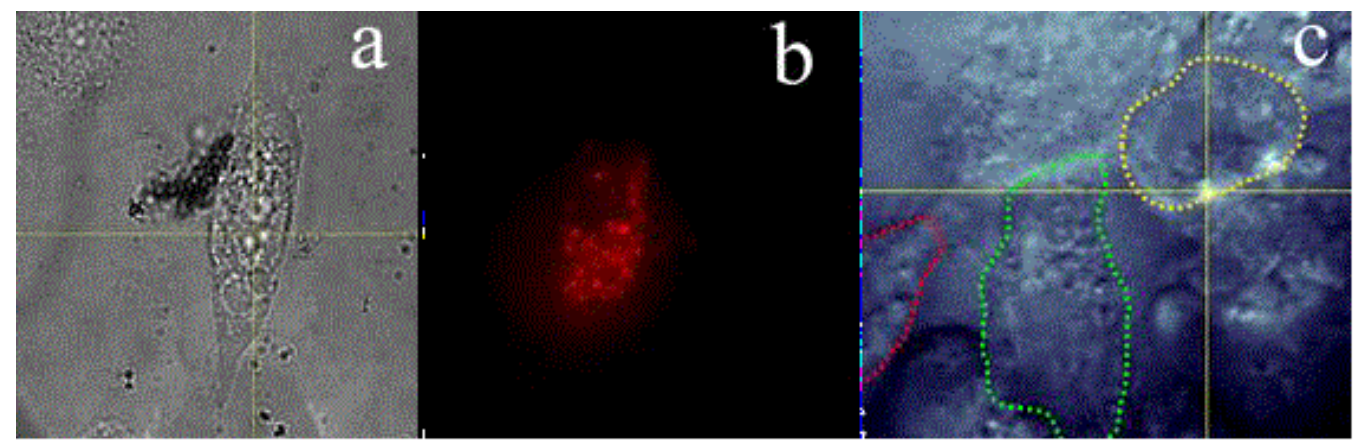

Figure 1. (a) White light microscopic and wide-field fluorescence images of (b) Se nanoparticles inside fibroblast cell - a snapshot from the movie demonstrating photostable in vitro fluorescence14 and (c) $\mathrm{ZnO}$ nanoparticles inside human skin keratinocyte cells, denoted by contours [15]

successfully integrated in one compound 12 or coated with some other materials to maintain photostability [13]. However, some recent studies indicate that metal oxide and semi-metalic NPs can be tracked in cells by their own fluorescence, see Figure $1[14,15]$. The fluorescence of NPs was observed in the red to near infrared wavelength range upon excitation with the visible green wavelength. The NPs were successfully internalized in fibroblast cells and did not show any adverse effects on cellular processes when monitored for extended time intervals. In both instances the experiments were performed at body temperature, relying only on NPs' intrinsic fluorescence. In this way, there are two essential advantages of this methodology. One is that there is no induced change of the chemistry or dynamics of these NPs. Most importantly, their fluorescence differs from the cells' auto-fluorescence, as long they are excited at wavelength longer than $500 \mathrm{~nm}$ (i.e., green or red laser). This combination of properties enables the efficient and safe use of these NPs depending on concentration $[14,15]$. Furthermore, these NPs are biodegradable making them ideal candidates for short-term bioimaging applications [16,17].

Thus, intrinsic fluorescence of metal-oxide and semi-metallic NPs at bio-suitable wavelengths of excitation and emission, can be observed, without the use of any additional tags. This fluorescence can be used to explore the biomedical properties of these NPs and make them attractive traceable therapeutic and diagnostic agents.

\section{References}

1. Wu J, Cao J, Han WQ, Janotti A, Kim HC (2012) Functional Metal Oxide Nanostructures. Springer Series in Materials Science.

2. Jagadish C, Pearton SJ (2011) Zinc Oxide Bulk, Thin Films and Nanostructures: Processing, Properties, and Applications. Elsevier.

3. Morfa AJ, Gibson BC, Karg M, Karle TJ, Greentree AD, et al. (2012) Single-photon emission and quantum characterization of zinc oxide defects. Nano Lett 12: 949-954. [Crossref]

4. Boyd R (2011) Selenium stories. Nat Chem 3: 570. [Crossref]
5. Rasmussen JW, Martinez E, Louka P, Wingett DG (2011) Zinc oxide nanoparticles for selective destruction of tumor cells and potential for drug delivery applications. Expert Opin Drug Deliv 7: 1063-1077. [Crossref]

6. Hajipour MJ, Fromm KM, Ashkarran AA, Jimenez de Aberasturi D, de Larramend IR, et al. (2012) Antibacterial properties of nanoparticles. Cell 30: 499-511. [Crossref]

7. Monici M (2005) Cell and tissue autofluorescence research and diagnostic applications. Biotechnol Annu Rev 11: 227-256. [Crossref]

8. Vahrmeijer AL, Hutteman M, van der Vorst JR, van de Velde CJH, Frangioni JV (2013) Image-guided cancer surgery using near-infrared fluorescence. Nat Rev Clin Oncol 10: 507-518. [Crossref]

9. Hayashi-Takanaka Y, Stasevich TJ, Kurumizaka H, Nozaki N, Kimura H (2014) Evaluation of chemical fluorescent dyes as a protein conjugation partner for live cell imaging. PLoS One 9: e106271. [Crossref]

10. Winnik FM, Maysinger D (2013) Quantum dot cytotoxicity and ways to reduce it. Acc Chem Res 46: 672-680. [Crossref]

11. Mochalin VN, Shenderova O, Ho D, Gogotsi Y (2011) The properties and applications of nanodiamonds. Nat Nanotechnol 7: 11-23. [Crossref]

12. Kaminska I, Sikora B, Fronc K, Dziawa P, Sobczak K, et al. (2013) Novel ZnO/MgO/ Fe2O3 composite optomagnetic nanoparticles. J Phys Condens Matter 25: 194105. [Crossref]

13. Weng B, Yang MQ, Zhang N, Xu YJ (2014) Toward the enhanced photoactivity and photostability of $\mathrm{ZnO}$ nanospheres via intimate surface coating with reduced graphene oxide. J Mater Chem A Mater 2: 9380-9389.

14. Khalid A, Tran PA, Norello R, Simpson DA, O'Connor AJ, et al. (2016) Tracking of selenium nanoparticles in-vitro using their intrinsic fluorescence. Nanoscale 8: 3376385. [Crossref]

15. Chung K, Karle TJ, Khalid A, Abraham AN, Shukla R, Gibson BC, et al. (2017) Roomtemperature single-photon emission from zinc oxide nanoparticle defects and their in vitro photostable intrinsic fluorescence. Nanophotonics 6: 269-278.

16. Zhou J, Xu NS, Wang ZL (2006) Dissolving Behavior and Stability of ZnO Wires in Biofluids: A Study on Biodegradability and Biocompatibility of $\mathrm{ZnO}$ Nanostructures. Adv Mater 18: 2432-2435.

17. Maiyo F, Singh M (2017) Selenium nanoparticles: potential in cancer gene and drug delivery. Nanomedicine 12: 1075-1089.

Copyright: (C2018 Tomljenovic-Hanic S. This is an open-access article distributed under the terms of the Creative Commons Attribution License, which permits unrestricted use, distribution, and reproduction in any medium, provided the original author and source are credited. 